\title{
A Software Framework for Efficient System-level Performance Evaluation of Embedded Systems
}

\author{
Joseph E. Coffland * Andy D. Pimentel \\ Dept. of Computer Science \\ University of Amsterdam \\ Kruislaan 403, 1098 SJ, Amsterdam \\ The Netherlands \\ \{jcofflan,andy\}@science.uva.nl
}

\section{Keywords}

Embedded systems, co-simulation, performance evaluation

\begin{abstract}
The Sesame environment provides modeling and simulation methods and tools for the efficient design space exploration of heterogeneous embedded multimedia systems. In this paper we describe the Sesame software system and demonstrate its capabilities using several examples. We show that Sesame significantly reduces model construction time through the use of modeling component libraries, hierarchy, and advanced model structure description features.
\end{abstract}

\section{INTRODUCTION}

Modern embedded systems, like those for media and signal processing, increasingly have a heterogeneous system architecture consisting of components in the range from fully programmable processor cores to dedicated hardware components. These systems often provide a high degree of programmability as they need to target a range of applications with varying demands. Such characteristics greatly complicate the system design, making it more important to have good tools available for exploring different design choices at early design stages.

In the context of the Artemis project [14], we are developing an architecture workbench which provides modeling and simulation methods and tools for efficient design space exploration of heterogeneous embedded multimedia systems. This workbench allows for rapid performance evaluation of different architecture designs, application to architecture mappings, and hardware/software partitionings at multiple levels of abstraction and for a wide range of multimedia applications.

This paper presents an overview of the software infrastructure of our prototype modeling and simulation environment, called Sesame

\footnotetext{
* Joseph Coffland currently operates as an independent software developer out of New York City and is founder of the SinaXe project see http://www.sinaxe.org/ for more information.
}

Permission to make digital or hard copies of all or part of this work for personal or classroom use is granted without fee provided that copies are not made or distributed for profi t or commercial advantage and that copies bear this notice and the full citation on the first page. To copy otherwise, to republish, to post on servers or to redistribute to lists, requires prior specifi c permission and/or a fee.

SAC 2003 Melbourne, Florida, USA

Copyright 2003 ACM 1-58113-624-2/03/03 ...\$5.00.
$[15,16]$, which is currently being developed in the Artemis project. More specifically, we will discuss the tools and language support provided by Sesame and explain how they facilitate efficient performance evaluation of embedded (media) systems.

The remainder of this paper is organized as follows. Section 2 shortly introduces Sesame and discusses its global infrastructure. In Section 3, we present the Y-chart Modeling Language (YML) which is used for describing the structure of application and architecture models in Sesame. Section 4 discusses the tools for application modeling and for executing these models, while Section 5 describes the framework for architecture modeling and simulation. Section 6 describes how application events are mapped onto architecture components. Work related to Sesame is discussed in Section 7. In Section 8, we present some results we have achieved with Sesame and briefly describe future work. Finally section 9 concludes the paper.

\section{THE SESAME ENVIRONMENT}

The Sesame modeling and simulation environment $[15,16]$ facilitates the performance analysis of embedded systems architectures in a way that directly reflects the Y-chart design approach [10]. In Y-chart based design, a designer studies the target applications, makes some initial calculations, and proposes an architecture. The performance of this architecture is then quantitatively evaluated and compared against alternative architectures. For such performance analysis, each application is mapped onto the architecture under investigation and the performance of each application-architecture combination is evaluated. The resulting performance numbers may inspire the designer to improve the architecture, restructure the application(s) or modify the mapping between the two.

In accordance to the Y-chart approach, Sesame recognizes separate application and architecture models within a system simulation. An application model describes the functional behavior of an application, including both computation and communication behavior. The architecture model defines architecture resources and captures their performance constraints. Essential in this modeling methodology is that an application model remain independent from architectural specifics, assumptions on hardware/software partitioning, and timing characteristics. As a result, a single application model can be used to exercise different hardware/software partitionings and can be mapped onto a range of architecture models, possibly representing different system architectures or simply modeling the same system architecture at various levels of abstraction. After explicitly mapping an application model onto an architecture model, they are co-simulated via trace-driven simulation and the 


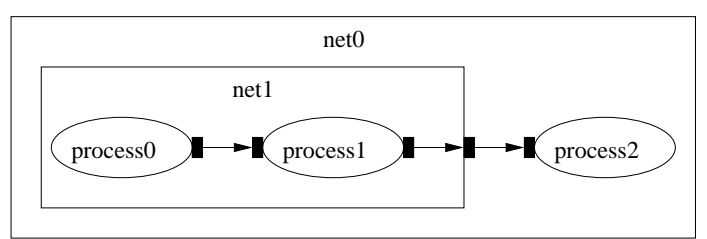

Figure 2: A process network example.

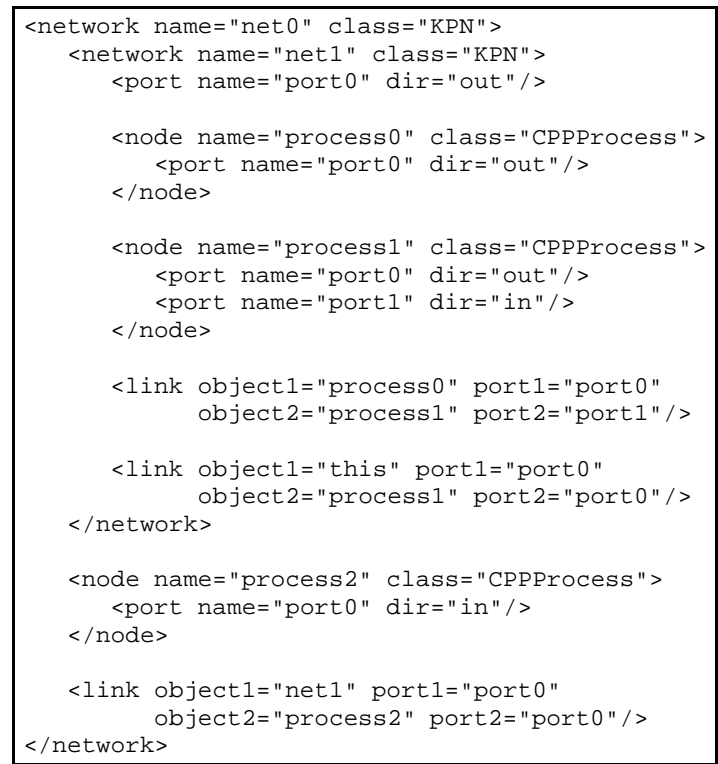

Figure 3: YML description of process network in Figure 2.

can be used. The script element takes no attributes. The text within a script element will be processed by the script interpreter in the order it appears in the YML file. YML attributes in name, class, or value elements that begin with a '\$' are evaluated as variables within the current context of the interpreter. At this point we do not have a good method for providing scope within Perl so users must be aware that all variables are global. This can be especially tricky when including external entities which contain a script as it is not immediately obvious which variables are modified by the included entity. An example is given together with the set example below.

\section{set}

Set elements further simplify the description of complex network structures by providing a for-loop like construct for the definition of YML structures. The set element requires three attributes init, cond, and loop whose values are interpreted as script. init is evaluated once at the beginning of the set processing. cond is evaluated at the beginning of every iteration of set processing. The value of cond is interpreted as a boolean. When it is false or 0 set processing stops. loop is evaluated once at the end of each iteration. When the following example YML is processed, five nodes named 'node0' through 'node4' will be created, each with a port named 'port0'.

\subsection{Additional features}

YML also benefits from the underlying XML language. By using XML parameter entities, YML libraries can be created. With library support and network hierarchy, model component and model component descriptions can be reused. In combination with the scripting feature, external entities can act as templates for complex structures. In Section 5, we describe how such templates are of great importance to flexible architectural modeling.

YML is a general purpose model description language and can be easily interfaced to any existing simulation tool either by translating YML to native description languages or by a direct interface to a simulator's internal data structures. Direct interfacing can be achieved using existing XML parsers alone or with assistance from YML programming interfaces.

\section{THE APPLICATION LAYER}

We model application behavior using Kahn process networks (KPNs), because they expose application parallelism, make communication explicit, and execute deterministicly [9]. It was previously shown in Section 3 how process networks are described in YML. To execute Kahn application models, we implemented (in $\mathrm{C}++$ ) a runtime system called PNRunner or Process Network Runner. This system does not directly name Kahn because it has been designed to allow for the addition of new interconnection classes that implement rules of other process network models which we may wish to support in the future. PNRunner reads a YML process network description and executes the described application model. The core of PNRunner is not concerned with how, where, or in what language processes are run. This is abstracted away through process loader classes. Processes may consist of $\mathrm{C}, \mathrm{C}++$, Java code, or even run on a remote machine. PNRunner also makes no assumptions about the type of data communicated between processes. From the internal view of PNRunner, communications consist of blocks of byte data. It is up to specific process loaders and the processes themselves to interpret the data. The class attribute of YML node elements tells PNRunner which process loader to use.

Currently, our main process loader is a C++ class loader which supports part of the YAPI interface [7]. YAPI was developed at Philips Research for application modeling with KPNs. YAPI describes KPNs completely in $\mathrm{C}++$ and therefore specifies the network structure implicit in the source code. It also provides applications with threading support and inter-process communication primitives. YAPI's implicit description of process network structure is redundant when using YML and therefore not supported in PNRunner. Sesame includes tools which ease conversion of YAPI applications to PNRunner applications.

Our C++ process loader recognizes a few special YML properties. Most importantly, the library and class properties tell the loader which shared library contains the process code and the name of the class in that library which implements the process. In support of the YAPI interface, which passes process parameters via the process constructor, we have two additional properties, namely carg and arg. carg's are constructor arguments and arg's are general arguments which can either be used in the constructor ar- 
guments or accessed by the process directly. arg's are passed at runtime unlike carg's which are fixed at compile time. Process classes are loaded via a generated stub. We have a separate tool to generate this stub code from YML descriptions. The following is a YML process description of a Video-Out application model process which originates from a M(otion)-JPEG application we studied in [15]. The corresponding auto-generated stub code follows.
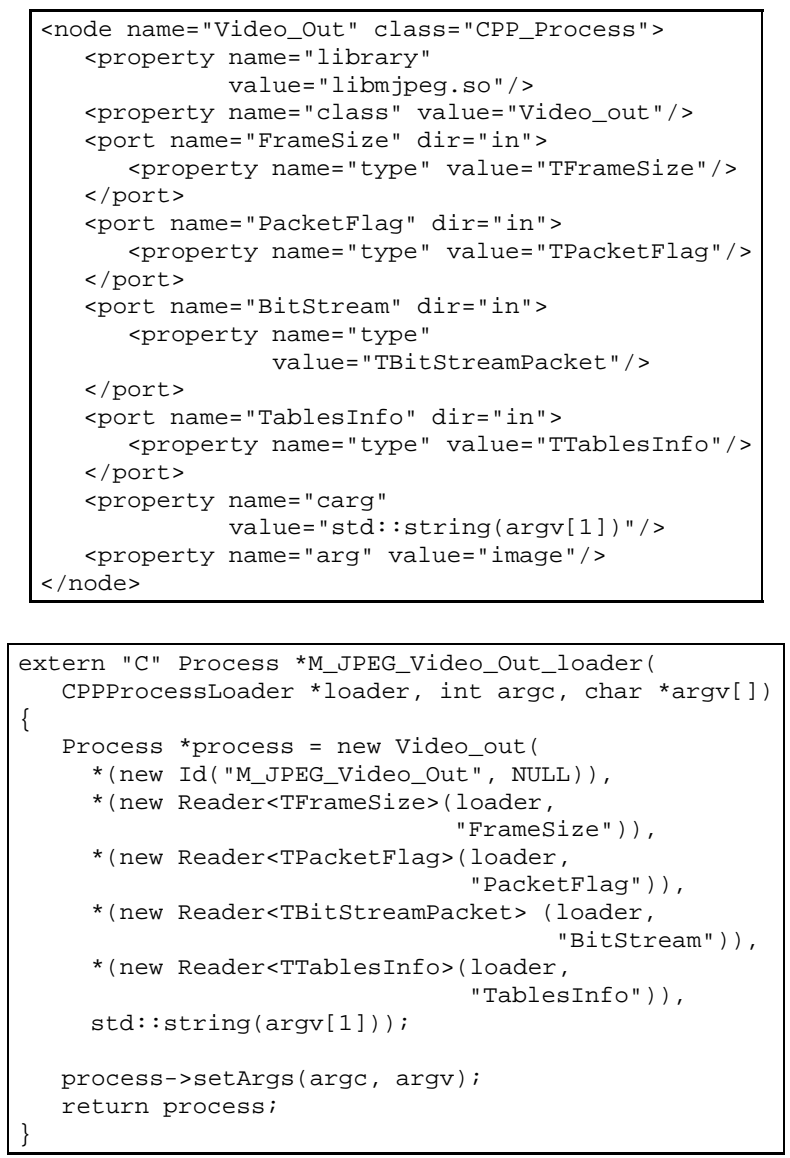

As can be seen above, the Video_out process (as are all PNRunner $\mathrm{C}++$ processes) is derived from the parent class Process giving it a common interface. The above example also shows another PNRunner YML property, namely the type port property. Like in YAPI, our interface uses C++ templated ports to control the datatype of transmitted data. If a link connects two ports of differing type, then an error will be generated. In the above example, the $\mathrm{C}++$ process loader calls the M_JPEG_Video_Out_loader function to get a pointer to a newly created Video_out process.

Application simulations can be analyzed in isolation, but PNRunner also supports the mapping of an application model onto an architecture model by providing processes with a trace-event API. The specific process loaders define exactly how processes access this interface. Communication trace events are automatically generated by PNRunner as data is read and written to process network communication channels. Processes must however explicitly signal execution events. To this end, our $\mathrm{C}++$ interface uses the YAPI approach. Running processes can emit execution trace events by calling the function execute ( $\operatorname{char} *$ ) and passing a string representing the execution event. Currently, PNRunner can output trace-event streams to files which, in UNIX systems, may be special files such as named pipes. More output options, such as a shared memory interface, will be considered as future work.

\section{THE ARCHITECTURE LAYER}

The Sesame architecture models, which simulate the timing consequences of the events generated by an application model, are implemented in the Pearl ${ }^{1}$ discrete-event simulation language [13]. This is a small but powerful object-based language which provides easy construction of (abstract) architecture models and fast simulation. It has a C-like syntax with a few additional primitives for simulation purposes. Architectures are modeled as communicating components (i.e., Pearl objects). Communication between components is performed using the special Pearl primitives '!' (synchronous communication) and '!!' (asynchronous communication). The Pearl runtime system accounts for simulated time implicitly as components block for communication events or explicitly as they model computation with the blockt primitive which blocks on the simulation clock.

To illustrate some of Pearl's primitives and to demonstrate the ease of modeling in Pearl, Figure 4 shows the code of a bus model. This model was used in a study in which we mapped the aforementioned M-JPEG application model onto a shared-memory multiprocessor architecture model [15]. The model simulates bus transactions at the granularity of message transfers of abstract data types. As Pearl is an object-based language and architecture components are modeled by objects, the code shown in Figure 4 embodies the class of bus objects.

The bus object has two object variables, mem and setup. These variables are initialized at the beginning of the simulation, and more specifically, at the instantiation of a bus object. The mem variable references the memory object that is connected to the bus, while the setup time of a connection on the bus is specified by setup. A bus object has two functions: load and store. The store function is not shown here since it is identical to the load function. The bus object uses the blockt ( ) primitive to wait for setup time units in order to account for the connection setup latency. The statement "mem ! load (nbytes, address)" calls the load function of the memory object mem by sending it a synchronous message. Since it is synchronous the bus has to wait until the memory has explicitly returned a reply message. The latter is done by the reply () primitive. In our example, the synchronous message passing also causes the virtual clock to advance in time, because the memory object accounts for the time it takes to retrieve the requested data before replying to the bus. After having received a reply from the memory object, the bus itself executes a

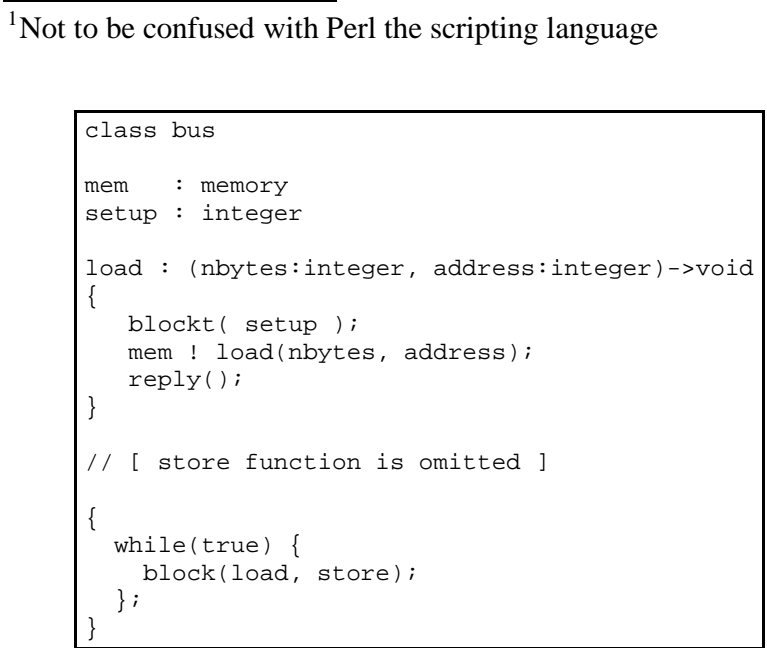

Figure 4: Pearl code for a bus object. 
requires that sometimes large numbers of port, process, and hardware component identifiers match up correctly in the event traces. Future enhancements to YML will remove this problem via a YML mapping description. In addition, this mapping description will be used to automatically generate the required mapping layer for a particular system simulation. The user's task will then be reduced from manually creating the mapping layer to simply writing a YML mapping description.

As a practical example of Sesame's effectiveness, we performed a number of case studies. In [15], we studied an M-JPEG application. Here we conducted experiments with a shared-memory multiprocessor architecture model. For this architecture, we evaluated different hardware-software partitionings, application to architecture mappings, processor speeds, and interconnect structures: bus, crossbar, and omega networks. With the Sesame software system, all this work including the application and architecture modeling, took less than one person-month.

Moreover, in [16], we studied different instances of the wellknown QR decomposition application. For this case study, we used QR application models that were also translated into VHDL [8]. This gave us the unique opportunity to compare our simulated performance measures to a real hardware implementation on an FPGA. The five different instances of the QR application we studied each expose a different degree of task parallelism. Our initial abstract architecture model yielded performance estimates that were on average $36 \%$ off with respect to the FPGA implementation. Following the Y-Chart design methodology, we refined our processor architecture model into several functional units and a control unit to better represent the FPGA implementation. As a result, we were able to come within $3.5 \%$ average case and $4.7 \%$ worst case of the FPGA performance results. This was done with less than 400 lines of Pearl code and around 400 lines of YML to describe both architecture and application models. It took Sesame about 16 seconds on a $333 \mathrm{Mhz}$ Sun Ultra 10 to perform the architecture simulations for all five application model instances in a batch.

\section{CONCLUSION}

With increasingly heterogeneous architectures and larger numbers of applications to support, embedded-systems tools like Sesame have become necessary to manage the complexity of design space exploration. With the Sesame software system we have created an embedded system co-simulation environment enabling developers to follow the Y-Chart design methodology. Changes can be made and the performance results evaluated quickly giving the designer more freedom to explore and discover an optimum design.

In this paper we have described the Sesame software system and demonstrated its effectiveness in modeling and simulating heterogeneous embedded multimedia system. We will continue to use Sesame as a vehicle for future research and to make this tool available for embedded system designers and researchers in the field. See http://sesamesim.sourceforge.net/ for the most up-to-date information regarding the Sesame project.

\section{Acknowledgments}

This research is supported by PROGRESS, the embedded systems research program of the Dutch organization for Scientific Research NWO, the Dutch Ministry of Economic Affairs and the Technology Foundation STW. We thank Frank Terpstra and Simon Polstra for their work on YML and PNRunner.

\section{REFERENCES}

[1] Cadence Design Systems, Inc., http://www.cadence.com/.
[2] Innoveda Inc., http://www.innoveda.com/.

[3] F. Balarin, E. Sentovich, M. Chiodo, P. Giusto, H. Hsieh, B. Tabbara, A. Jurecska, L. Lavagno, C. Passerone, K. Suzuki, and A. Sangiovanni-Vincentelli. Hardware-Software Co-design of Embedded Systems - The POLIS approach. Kluwer Academic Publishers, 1997.

[4] T. Bray, J. Paoli, C. M. Sperberg-McQueen, and E. Maler. Extensible Markup Language (XML) 1.0 Second Edition, October 2000.

[5] J. Buck, S. Ha, E. A. Lee, and D. G. Messerschmitt. Ptolemy: A framework for simulating and prototyping heterogeneous systems. Int. Journal of Computer Simulation, 4:155-182, Apr. 1994.

[6] A. Cohen, D. Genius, A. Kortebi, Z. Chamski, M. Duranton, and P. Feautrier. Multi-periodic process networks: Prototyping and verifying stream-processing systems. In Proc. of EuroPar'02, Aug. 2002.

[7] E. A. de Kock, G. Essink, W. J. M. Smits, P. van der Wolf, J. Y. Brunel, W. M. Kruijtzer, P. Lieverse, and K. A. Vissers. Yapi: Application modeling for signal processing systems. In Proc. of the Design Automation Conference, pages 402-405, June 2000.

[8] T. Harris, R. Walke, B. Kienhuis, and E. Deprettere. Compilation from matlab to process networks realized in fpga. In Proc. of the 35 Asilomar conference on Signals, Systems, and Computers, Nov. 2001.

[9] G. Kahn. The semantics of a simple language for parallel programming. In Proc. of the IFIP Congress 74, 1974.

[10] B. Kienhuis, E. F. Deprettere, K. A. Vissers, and P. van der Wolf. An approach for quantitative analysis of application-specific dataflow architectures. In Proc. of the Int. Conf. on Application-specific Systems, Architectures and Processors, July 1997.

[11] E. A. Lee and S. Neuendorffer. MoML - a Modeling Markup Language in XML, version 0.4. Technical Report UCB/ERL M00/8, Electronics Research Lab, University of California, Berkeley, March 2000.

[12] P. Lieverse, P. van der Wolf, E. F. Deprettere, and K. A. Vissers. A methodology for architecture exploration of heterogeneous signal processing systems. Journal of VLSI Signal Processing for Signal, Image and Video Technology, 29(3):197-207, Nov. 2001. Special issue on SiPS'99.

[13] H. Muller. Simulating computer architectures. $\mathrm{PhD}$ thesis, Dept. of Computer Science, Univ. of Amsterdam, Feb. 1993.

[14] A. D. Pimentel, P. Lieverse, P. van der Wolf, L. O. Hertzberger, and E. F. Deprettere. Exploring embedded-systems architectures with Artemis. IEEE Computer, 34(11):57-63, Nov. 2001.

[15] A. D. Pimentel, S. Polstra, F. P. Terpstra, A. W. van Halderen, J. E. Coffland, and L. O. Hertzberger. Towards efficient design space exploration of heterogeneous embedded media systems. In Embedded Processor Design Challenges: Systems, Architectures, MOdeling, and Simulation, pages 57-73. Springer, LNCS 2268, 2002.

[16] A. D. Pimentel, F. P. Terpstra, S. Polstra, and J. E. Coffland. Modeling of intra-task parallelism in Sesame. In Proc. of the 2nd Int. Workshop on Systems, Architectures, MOdeling, and Simulation (SAMOS-II), pages 1-16, July 2002. 